\title{
FIRST COEFFICIENT DOMAINS AND IDEALS OF REDUCTION NUMBER ONE
}

\author{
William Heinzer ${ }^{1}$, Bernard Johnston and David Lantz
}

Department of Mathematics, Mathematical Sciences Building, Purdue University, West Lafayette, IN 47907. E-mail: heinzer@math.purdue.edu

Department of Mathematics, Florida Atlantic University, Boca Raton, FL 33431. E-mail: johnston@cse.fau.edu

Department of Mathematics, Colgate University, 13 Oak Drive, Hamilton, NY 133461398. E-mail: dlantz@colgateu.bitnet

\section{Introduction.}

(1.1) Let $(R, \mathbf{m})$ be a 2-dimensional regular local domain with $R / \mathbf{m}$ infinite, and let $I$ be an m-primary ideal. We write the Hilbert polynomial, measuring the lengths $\lambda\left(R / I^{n}\right)$ for sufficiently large $n$, in the form:

$$
P_{I}(n)=e_{0}(I)\left(\begin{array}{c}
n+1 \\
2
\end{array}\right)-e_{1}(I)\left(\begin{array}{l}
n \\
1
\end{array}\right)+e_{2}(I)
$$

We consider a sequence of ideals $I \subseteq \widetilde{I} \subseteq I_{\{1\}} \subseteq I^{\prime}$, where $I^{\prime}$ denotes the integral closure of $I$. It is well known that $I^{\prime}$ is the largest ideal containing $I$ and having the same multiplicity as $I$, i.e., for which $e_{0}\left(I^{\prime}\right)=e_{0}(I)$. The ideal $\widetilde{I}=\bigcup_{n=1}^{\infty}\left(I^{n+1}: I^{n}\right)$, the "Ratliff-Rush ideal associated to $I$," is the largest ideal containing $I$ and having the same Hilbert polynomial as $I$. (We give a fuller explanation of our terminology below.) It is shown by Shah in [Sh1] that there exists an ideal $I_{\{1\}}$, the "first coefficient ideal" or " $e_{1}$-ideal"

\footnotetext{
${ }^{1}$ The first author would like to acknowledge the support of NSF Grant DMS-9101176, and the third author the hospitality of Purdue University, while this work was done.
} 
associated to $I$, that is uniquely maximal among those containing $I$ for which the first two coefficients of their Hilbert polynomials are equal to those of $I$ : $e_{0}(I)=e_{0}\left(I_{\{1\}}\right)$ and $e_{1}(I)=e_{1}\left(I_{\{1\}}\right)$. By Narita $[\mathrm{N}]$, the third coefficients, the constant terms of the Hilbert polynomials, satisfy $e_{2}(I) \geq e_{2}\left(I_{\{1\}}\right) \geq 0$. Thus, if $e_{2}(I)=0$, then $\widetilde{I}=I_{\{1\}}=(\widetilde{I})_{\{1\}}$, i.e., $\widetilde{I}$ is a "first coefficient ideal" or "e $e_{1}$-ideal."

An example illustrating these ideals is:

Example 1.2. Let $R=k[x, y]_{(x, y)}$, where $k$ is a field and $x, y$ are indeterminates, and set

$$
I=\left(x^{16}, x^{12} y^{4}, x^{10} y^{6}, x^{6} y^{10}, x^{4} y^{12}, y^{16}\right) R
$$

Then $\widetilde{I}=\left(x^{16}, x^{12} y^{4}, x^{10} y^{6}, x^{8} y^{8}, x^{6} y^{10}, x^{4} y^{12}, y^{16}\right) R, I_{\{1\}}=\left(x^{2}, y^{2}\right)^{8} R$, and $I^{\prime}=(x, y)^{16} R$. Thus, $I \subset \widetilde{I} \subset I_{\{1\}} \subset I^{\prime}$ (strict containments). The Hilbert polynomials of $I$ (or of $\widetilde{I}$ ) and of $I_{\{1\}}$ are respectively

$$
256\left(\begin{array}{c}
n+1 \\
2
\end{array}\right)-112\left(\begin{array}{c}
n \\
1
\end{array}\right)+8 \quad \text { and } \quad 256\left(\begin{array}{c}
n+1 \\
2
\end{array}\right)-112\left(\begin{array}{c}
n \\
1
\end{array}\right)
$$

In particular, $\lambda\left(I_{\{1\}} / \widetilde{I}\right)=e_{2}(I)$.

This paper arose from our interest in whether an $e_{1}$-ideal $I$ primary for the maximal ideal in a 2-dimensional regular local domain satisfies $e_{2}(I)=0$. There appear in the literature several statements equivalent to the conditions that $I=\widetilde{I}$ and $e_{2}(I)=0$. Among them are: (1) $I$ has reduction number at most one, i.e., if $J$ is a minimal reduction of $I$, then $J I=I^{2}$. (2) $\lambda(R / I)=$ $e_{0}(I)-e_{1}(I)$. (3) The Rees algebra $R[I t]$ is Cohen-Macaulay. It is shown in [HJLS] that all sufficiently high powers of $I$ are $e_{1}$-ideals iff the blowup $\mathcal{B}(I)=\operatorname{Proj}(R[I t])$ is Cohen-Macaulay, a condition formally weaker than 
(3). We show in Example 5.4 below the existence of an m-primary ideal $I$ of $R$ such that all powers of $I$ are $e_{1}$-ideals, but $R[I t]$ is not Cohen-Macaulay.

(1.3) In studying the association $I \mapsto I_{\{1\}}$, we have found it useful to attach to $I$ a certain 1-dimensional semilocal domain $D$, the "first coefficient domain" of $I$, that plays a role for this association similar to that played by the intersection of the Rees valuation domains of $I$ for integral closure: $I^{n} D \cap R=\left(I^{n}\right)_{\{1\}}$ for each positive integer $n$. The paper [HJLS] contains some information on the structure of $e_{1}$-ideals and other "coefficient ideals." In particular, it is from Theorem 3.17 of that paper (repeated as Theorem 2.10(a) below) that we deduce the equation $I^{n} D \cap R=\left(I^{n}\right)_{\{1\}}$. In the present paper we establish some basic facts about such domains and prove several properties of $e_{1}$-ideals and ideals of reduction number at most one (usually primary for the maximal ideal in a local domain).

Before we describe the contents of the paper in more detail, we establish some basic notation and terminology.

(1.4) We denote the length of the $R$-module $A$ by $\lambda_{R}(A)$, omitting the subscript when the context allows. If $I$ is an ideal in a Noetherian ring $R$ for which $\operatorname{dim}(R / I)=0$, then the Hilbert function of $I, H_{I}(n):=\lambda\left(R / I^{n}\right)$, is, for all sufficiently large values of the positive integer $n$, a polynomial in $n$ of degree $d:=\operatorname{dim}(R)$, the Hilbert polynomial of $I$, which we denote by $P_{I}(n)$. If $P_{I}$ is written in terms of binomial coefficients:

$$
P_{I}(n)=e_{0}(I)\left(\begin{array}{c}
n+d-1 \\
d
\end{array}\right)-e_{1}(I)\left(\begin{array}{c}
n+d-2 \\
d-1
\end{array}\right)+\cdots+(-1)^{d} e_{d}(I)
$$

then the coefficients $e_{i}=e_{i}(I)$ are integers, the Hilbert coefficients of $I$. The leading coefficient $e_{0}(I)=e(I)$ is the multiplicity of $I$.

(1.5) Let $I$ be an $\mathbf{m}$-primary ideal in a quasi-unmixed local domain $(R, \mathbf{m})$ 
of dimension $d>0$ with $R / \mathbf{m}$ infinite. It is shown by Shah in [Sh1] that, for each integer $m$ in $\{0,1, \ldots, d\}$, there is a unique largest ideal $I_{\{m\}}$ containing $I$ for which $e_{i}\left(I_{\{m\}}\right)=e_{i}(I)$ for $i=0, \ldots, m$. (Shah's results are valid in the case of rings with zero-divisors, but in the present paper we choose to restrict to the domain case.) In [HJLS] we call $I_{\{m\}}$ the $m$-th coefficient ideal associated to $I$ or the $e_{m}$-ideal associated to $I$; and if $I_{\{m\}}=I$, then we call $I$ an $m$-th coefficient ideal or an $e_{m}$-ideal. In particular, $I_{\{0\}}=I^{\prime}$ by $[\operatorname{Re} 1$, Theorem 3.2, page 16]; and $I_{\{d\}}=\widetilde{I}$, the Ratliff-Rush ideal associated to $I$, is the largest ideal for which $(\widetilde{I})^{n}=I^{n}$ for sufficiently large positive integers $n$. It is immediate that

$$
I \subseteq I_{\{d\}} \subseteq I_{\{d-1\}} \subseteq \cdots \subseteq I_{\{1\}} \subseteq I_{\{0\}}=I^{\prime}
$$

Most of Section 2 is devoted to establishing our notation and basic facts on blowups $\mathcal{B}(I)$ of ideals $I$ and other models that we use in the sequel. Let $(R, \mathbf{m})$ be a $d$-dimensional, normal, analytically unramified local domain with $R / \mathbf{m}$ infinite. In Theorem 2.13 , we show that each projective model $X$ over $\operatorname{Spec}(R)$ such that $\mathcal{B}(I) \preceq X \preceq B(I)^{\prime}$ is of the form $X=\mathcal{B}(J)$ for some ideal $J$ integral over a power of $I$.

Again, let $(R, \mathbf{m})$ be a quasi-unmixed, analytically unramified local domain with infinite residue field, and let $m \leq \operatorname{dim}(R)$. Given an mprimary ideal $I$ of $R$, we define in Section 3 the $m$-th coefficient domain $D_{m}$ of $I$, a semilocal domain of dimension at most $m$; and we observe that $I^{n} D_{m} \cap R=\left(I^{n}\right)_{\{m\}}$. Specializing to first coefficient domains, we display several methods of realizing them; and we show in Theorem 3.12 that, if in addition $R$ is normal, then a domain birational and integral over the first coefficient domain of an ideal $I$ is the first coefficient domain of an ideal 
integral over a power of $I$.

In Section 4, we explore the similarities and relationship between the concepts of ideals of reduction number at most one and first coefficient ideals. We show that, under reasonable hypotheses, the intersections of both these classes with the family of ideals having a fixed common reduction are closed under intersection. Suppose $(R, \mathbf{m})$ is a 2-dimensional, Cohen-Macaulay, analytically unramified local domain with $R / \mathbf{m}$ infinite, $I=(a, b) R$ is an m-primary ideal, and $D$ is its first coefficient domain. Then for an $e_{1}$-ideal $J$ integral over $I$, we show in Theorem 4.8 that $e_{1}(J)=\lambda_{D}(E / D)$ where $E$ is the first coefficient domain of $J$, and that $J$ has reduction number at most one iff $J D$ does.

In Section 5 we provide general and specific examples, in the context of a 2-dimensional regular local domain, of the concepts we have introduced earlier. In particular, Example 5.4 is that of an ideal of reduction number 2, all of whose powers are $e_{1}$-ideals.

\section{Preliminaries: Blowups of ideals and other models.}

We abbreviate "regular local domain" by RLR and "discrete rank-one valuation domain" by DVR. Our use of the terms "local" and "semilocal" includes the condition that the ring is Noetherian. We say that a semilocal integral domain $S$ "birationally dominates" a local domain $(R, \mathbf{m})$ if $S$ contains $R, S$ is contained in the field of fractions of $R$, and each maximal ideal of $S$ contains $\mathbf{m}$.

We record in this section the conventions and results concerning models and related matters that we use below.

(2.1) Let $R$ be a Noetherian domain and $X$ be a birational model over 
$R$, i.e., a finite union of spectra (regarded as a family of local domains) of finitely generated $R$-algebras between $R$ and its field of fractions $K$ such that, for any valuation domain $V$ between $R$ and $K$, there is at most one domain in $X$ that is dominated by $V$. The model $X$ is complete over $R$ if every such $V$ dominates an element of $X$.

(2.2) For an ideal $I$ of $R$, we will abbreviate the contraction $\bigcap\{I S \cap R$ : $S \in X\}$ to $R$ of the extension of $I$ to (the local domains in) $X$ by $I X \cap R$. We also speak of the elements of $X$ as prime ideals by identifying the local domain with its maximal ideal. (Of course, two local domains may share the same maximal ideal, but this is not true of two local domains on the same model.)

(2.3) For an ideal $I$ of $R$ and a local domain $S$ in $X$, if the maximal ideal of $S$ is an associated prime (respectively, a prime minimal over) the extension $I S$ of $I$ to $S$, we call $S$ an associated prime (respectively, a minimal prime) of $I X$. There are only finitely many local domains, say $S_{1}, S_{2}, \ldots, S_{n}$, in $X$ that are associated primes of $I X$, and we have the equality $I X \cap R=$ $I S_{1} \cap I S_{2} \cap \ldots \cap I S_{n} \cap R$.

(2.4) An ideal $J$ contained in an ideal $I$ is a reduction of $I$ if there is a positive integer $n$ for which $J I^{n}=I^{n+1}$. This is equivalent to saying that $I$ is integral over $J$, i.e., that each element $a$ of $I$ satisfies an equation of the form $a^{n}+b_{1} a^{n-1}+\cdots+b_{n-1} a+b_{n}=0$ where $b_{j} \in J^{j}$ for each $j$ in $\{1, \ldots, n\}$. The largest ideal $J^{\prime}$ of $R$ that is integral over $J$ (i.e., of which $J$ is a reduction) is the integral closure of $J$. An ideal $I$ has at least one minimal reduction $J$ (with respect to inclusion); the smallest $n$ for which $J I^{n}=I^{n+1}$, as $J$ varies over the minimal reductions of $I$, is the reduction number of $I$. If $(R, \mathbf{m})$ is local with infinite residue field, then the minimal 
number of generators of a minimal reduction of $I$ is equal to the dimension of the ring $\bigoplus_{n=0}^{\infty} I^{n} / \mathbf{m} I^{n}$; this dimension is bounded below by the height of $I$ and above by the dimension of $R$.

(2.5) For an ideal $I$ of $R$, the blowup $\mathcal{B}(I)$ of $I$, defined by

$$
\begin{aligned}
\mathcal{B}(I) & =\bigcap\{\operatorname{Spec}(R[I / a]: a \in I-0\} \\
& =\left\{R[I / a]_{P}: a \in I-0, P \text { prime in } R[I / a]\right\},
\end{aligned}
$$

is the model over $R$ consisting of the family of all local domains $S$ between $R$ and its field of fractions that are minimal with respect to domination among those in which the extension of $I$ is principal. The elements $a$ in this definition need not vary over all the nonzero elements of $R ; \mathcal{B}(I)$ is also the union of the spectra of the rings $R[I / a]$ as $a$ varies over a generating set of $I$ or even over a generating set for a reduction of $I$. A model $X$ is the blowup of an ideal of $R$ iff it is projective (cf. $[\mathrm{ZS}])$ and birational over $R$.

(2.6) If $X, Y$ are models complete and birational over $R$, we say $X d o m i$ nates $Y$ and write $Y \preceq X$ if every local domain in $Y$ is dominated by a local domain in $X$. If $I \subseteq J$ are ideals of $R$ such that $J$ is integral over $I$, then $\mathcal{B}(I) \preceq \mathcal{B}(J)$.

(2.7) For a birational model $X$ over $R$, we will frequently consider two related families of local domains: the normalization $X^{\prime}$ of $X$, consisting of the union of the spectra of the integral closures of the local domains in $X$; and the "S $S_{2}$-ification" $X^{(1)}$ of $X$, consisting of the union of the spectra of the rings $S^{(1)}=\bigcap\left\{S_{\mathbf{p}}: \mathbf{p}\right.$ ht-1 prime in $\left.S\right\}$ as $S$ varies over $X$. Without additional hypotheses, $X^{\prime}$ and $X^{(1)}$ may not be models over $R$, because a local domain on $X^{\prime}$ or $X^{(1)}$ may not be a localization of a finitely generated $R$-algebra. But suppose $R$ is a quasi-unmixed analytically unramified local 
domain. Then for a model $X$ complete and birational over $R, X^{(1)}$ and $X^{\prime}$ are complete models over $R$ and $X \preceq X^{(1)} \preceq X^{\prime}$; and if $X$ is projective over $R$, then $X^{\prime}$ is also projective over $R$. It is well known that $I^{\prime}=I \mathcal{B}(I)^{\prime} \cap R$; we also have $\widetilde{I}=I \mathcal{B}(I) \cap R$ [HLS, Fact 2.1] and, if in addition $R$ has infinite residue field, then $I_{\{1\}}=I \mathcal{B}(I)^{(1)} \cap R$ [HJLS, Theorem 3.17].

(2.8) The model denoted $\mathcal{B}(I)^{(1)}$ in the present paper may properly dominate the model denoted $\mathcal{B}(I)^{(1, I)}$ in [HJLS] (and occasionally abbreviated to $\mathcal{B}(I)^{(1)}$ there), but the sets of associated primes of $I \mathcal{B}(I)^{(1)}$ and $I \mathcal{B}(I)^{(1, I)}$ are both equal to the set of one-dimensional associated primes of $I \mathcal{B}(I)$, so the contractions $I \mathcal{B}(I)^{(1, I)} \cap R$ and $I \mathcal{B}(I)^{(1)} \cap R$ are equal. In general, $\mathcal{B}(I)^{(1)}=\mathcal{B}(I)^{(1, I)}$ if and only if $R_{\mathbf{p}}$ satisfies Serre's condition $S_{2}$ for each prime $\mathbf{p}$ of $R$ not containing $I$.

(2.9) In studying the coefficient ideals $I_{\{m\}}$ of $I$ defined in (1.5) for $m>1$, it is useful to consider, for $S$ a Noetherian domain, certain rings between $S$ and $S^{(1)}$ in which the associated primes of principal ideals are of height no greater than $m$. We set

$$
S^{(m)}:=\bigcap\left\{S_{\mathbf{p}}: \mathbf{p} \text { is a prime in } S, \operatorname{ht}(\mathbf{p}) \leq m\right\}
$$

and, for a model $X$ complete and birational over $R$,

$$
X^{(m)}:=\bigcup\left\{\operatorname{Spec}\left(S^{(m)}\right): S \in X\right\} .
$$

Assume that $R$ is universally catenary and that finitely generated birational $R$-algebras have finitely generated integral closure (if $R$ is local, then these conditions are equivalent to: $R$ is quasi-unmixed and analytically unramified), and let $d:=\operatorname{dim}(R)$. Then for a birational model $X$ complete over $R$, the sets $X^{(m)}$ are also complete models over $R$ and

$$
X=X^{(d)} \preceq X^{(d-1)} \preceq \cdots \preceq X^{(1)} \preceq X^{\prime} .
$$


Suppose $X$ dominates the blowup of an ideal $I$ of $R$. Then the model that was denoted $X^{(m, I)}$ in [HJLS] is dominated by $X^{(m)}$; though this domination may be proper, we have as in (2.8) above that the associated primes of the extension of $I$ are the same in these two models, and so $I X^{(m, I)} \cap R=$ $I X^{(m)} \cap R$. In particular, if in addition $R$ is local with infinite residue field and $X=\mathcal{B}(I)$, then by [HJLS, Theorem 3.17], this common contraction is the associated $e_{m}$-ideal $I_{\{m\}}$ of $I$ described in (1.5). For this reason and the fact that $I \mathcal{B}(I)^{\prime} \cap R=I^{\prime}=I_{\{0\}}$, and for later notational convenience, we set $\mathcal{B}(I)^{(0)}:=\mathcal{B}(I)^{\prime}$. We remark, generalizing the statement in (2.8), that $\mathcal{B}(I)^{(m)}=\mathcal{B}(I)^{(m, I)}$ iff each associated prime of a principal ideal in $R$ that does not contain $I$ has height at most $m$.

We restate Theorem 3.17 and Proposition 1.12 of [HJLS], because they are frequently used in the present paper, for instance in Theorem 2.13 below. The last assertion in part (b) follows from [Re2, Theorem 1.4].

Theorem 2.10. (a) Let $(R, \mathbf{m})$ be a d-dimensional quasi-unmixed analytically unramified local domain with $R / \mathbf{m}$ infinite, let $I$ be an $\mathbf{m}$-primary ideal, and let $k \in\{1, \ldots, d\}$. Then for each positive integer $n,\left(I^{n}\right)_{\{k\}}=$ $I^{n} \mathcal{B}(I)^{(k, I)} \cap R$. Moreover, for all sufficiently large integers $n, \mathcal{B}(I)^{(k, I)}$ is the blowup of $\left(I^{n}\right)_{\{k\}}$, and all the powers of $\left(I^{n}\right)_{\{k\}}$ are $e_{k}$-ideals.

(b) If $R$ is a Noetherian domain and $I$ is a nonzero ideal of $R$, then for each positive integer $n,\left(I^{n}\right)^{\prime}=I^{n} \mathcal{B}(I)^{\prime} \cap R$. If in addition $(R, \mathbf{m})$ is a normal, analytically unramified local domain, and I is $\mathbf{m}$-primary, then for all sufficiently large integers $n, \mathcal{B}(I)^{\prime}$ is the blowup of $\left(I^{n}\right)^{\prime}$, and all the powers of $\left(I^{n}\right)^{\prime}$ are integrally closed.

(2.11) Because we want to make use of the connection between coefficient 
ideals and models as described in Theorem 2.10(a) often in the present paper, when we discuss coefficient ideals or their associated models, we assume that $(R, \mathbf{m})$ is a quasi-unmixed analytically unramified local domain with infinite residue field, though we will often repeat these hypotheses in the statements of results.

Questions 2.12. (1) If $R=k[[x, y]]$ and $I$ is generated by monomials, need $I_{\{1\}}$ be as well?

(2) If $I$ is an $e_{1}$-ideal, must $I^{2}$ also be?

(3) If $I$ is an $e_{1}$-ideal, must $I^{n}$ also be for all large $n$ ?

(4) Suppose $I, J$ are $\mathbf{m}$-primary Ratliff-Rush ideals in a 2-dimensional $\operatorname{RLR}(R, \mathbf{m})$, having the same integral closure and the same blowup. Is this common blowup also the blowup of $I+J$ ? Must $I=J$ ? These questions are interesting even in the case where the common integral closure is a power of m. (Cf. Example 3.14.)

Theorem 2.13. Suppose $(R, \mathbf{m})$ is a d-dimensional, normal, analytically unramified, local domain with $R / \mathbf{m}$ infinite and $I$ is an $\mathbf{m}$-primary ideal in R. Then the set of projective models $X$ over $R$ such that $\mathcal{B}(I) \preceq X \preceq \mathcal{B}(I)^{\prime}$ satisfies the ascending chain condition with respect to domination. Moreover, for $0 \leq q<p \leq d$, if $\mathcal{B}(I)^{(p)} \preceq X \preceq \mathcal{B}(I)^{(q)}$, then there exists a positive integer $n$ and an ideal $J$ of $R$ such that $\left(I^{n}\right)_{\{p\}} \subseteq J \subseteq\left(I^{n}\right)_{\{q\}}$ and $X=\mathcal{B}(J)$. In particular, each projective model $X$ over $\operatorname{Spec}(R)$ such that $\mathcal{B}(I) \preceq X \preceq$ $B(I)^{\prime}$ is of the form $X=\mathcal{B}(J)$ for some ideal $J$ integral over a power of $I$.

Proof. Note that, since we have assumed that $R$ is normal, the models $\mathcal{B}(I)^{(k, I)}$ of Theorem 2.10 are equal to the models $\mathcal{B}(I)^{(k)}$ here. We first 
prove the following version of the first assertion: The set

$$
\left\{\mathcal{B}(J): J \text { an ideal, and } \exists n \in \mathbf{N} \text { such that } I^{n} \subseteq J \subseteq\left(I^{n}\right)^{\prime}\right\}
$$

satisfies the ascending chain condition under domination. This version is shown in the proof of Theorem 3.17 of [HJLS], although not explicitly stated there. We briefly recall the proof. Let $\left(a_{1}, \ldots, a_{d}\right) R$ be a reduction of $I$; then for any ideal $J$ between $I^{n}$ and $\left(I^{n}\right)^{\prime},\left(a_{1}^{n}, \ldots, a_{d}^{n}\right) R$ is a reduction of $J$, and $R\left[I / a_{i}\right]=R\left[I^{n} / a_{i}^{n}\right] \subseteq R\left[J / a_{i}^{n}\right] \subseteq R\left[I / a_{i}\right]^{\prime} ;$ since $\mathcal{B}(I), \mathcal{B}(J), \mathcal{B}(I)^{\prime}$ are respectively the unions of the spectra of the rings $R\left[I / a_{i}\right], R\left[J / a_{i}^{n}\right], R\left[I / a_{i}\right]^{\prime}$, it suffices to show that the collection of domains between $R\left[I / a_{i}\right]$ and $R\left[I / a_{i}\right]^{\prime}$ satisfies the ascending chain condition with respect to inclusion. And this collection does satisfy ACC, because the assumption that $R$ is analytically unramified guarantees that $R\left[I / a_{i}\right]^{\prime}$ is a finite $R\left[I / a_{i}\right]$-module by Rees's Theorem [Re2, Theorem 1.5, page 27].

Now we prove the full strength of the first assertion by proving the second: Assume by way of contradiction that the second assertion is false. Note that, in particular, the ascending chain condition holds for the family of models

$$
\left\{\mathcal{B}(J): J \text { an ideal, and } \exists n \in \mathbf{N} \text { such that }\left(I^{n}\right)_{\{p\}} \subseteq J \subseteq\left(I^{n}\right)_{\{q\}}\right\}
$$

Thus, in view of Theorem 2.10, by replacing $I$ by some ideal integral over one of its powers, we may assume that $\mathcal{B}(I)=\mathcal{B}(I)^{(p)}$ and that there is no $\mathcal{B}(J)$ in the above family such that $\mathcal{B}(I) \prec \mathcal{B}(J) \preceq X$.

Since every projective model birational over $\operatorname{Spec}(R)$ is the blowup of some ideal of $R$, we have $X=\mathcal{B}(K)$ for some ideal $K$ of $R$. We have assumed that $\mathcal{B}(I) \prec \mathcal{B}(K)$, so there is a prime $\mathbf{q}$ on $\mathcal{B}(I)$ that is not on $\mathcal{B}(K)$. Since $R / \mathbf{m}$ is infinite, there is an element $a$ of $I$ for which all of the following 
finitely many prime ideals $\mathbf{p}$ are on the same affine piece $S:=R[I / a]$ of $\mathcal{B}(I):(1) \mathbf{p}$ is an associated prime of $I \mathcal{B}(I) ;(2) \mathbf{p}$ is the contraction to $\mathcal{B}(I)$ of an associated prime of $I \mathcal{B}(K)$; or $(3) \mathbf{p}$ is the prime $\mathbf{q}$ of $\mathcal{B}(I)$ chosen above not on $\mathcal{B}(K)$.

Since $K S^{(q)}$ is a locally principal ideal of $S^{(q)}, \operatorname{Spec}\left(S^{(q)}\right)$ dominates the blowup $\mathcal{B}(K S)$ of $K S$ over $S$. (If $U$ is any local ring of $\mathcal{B}(K S)$, then it must be dominated by some valuation $\operatorname{ring} V$, which must in turn contain $S^{\prime}$ and hence $S^{(q)}$. Consequently $V$ must have a center on $S^{(q)}$ which in turn must dominate the unique center $U$ of $V$ on $\mathcal{B}(K S)$.) Hence by Chevalley's Theorem [EGA, Chap II, (6.7.1), p.136], $\mathcal{B}(K S)$ is an affine blowup over $S$, i.e., $\mathcal{B}(K S)=\operatorname{Spec}(T)$ where $T$ is a domain between $S$ and $S^{(q)}=R[I / a]^{(q)}$.

Since $I$ extends to a locally principal ideal on these models, $I$ and any power of $I$ have the same associated primes on these models. Thus, by (2.3), $I^{n} S \cap R=I^{n} \mathcal{B}(I) \cap R=\widetilde{I^{n}}$ and $I^{n} T \cap R=I^{n} \mathcal{B}(K) \cap R$, the latter because, by our choice of $S, \mathcal{B}(K S)$ contains all the associated primes of $I \mathcal{B}(K)$. We have $S<T \subseteq S^{(q)} \subseteq S^{\prime} \subseteq R[1 / a]$, the last inclusion because $R$ is normal, so that the local domains on $\mathcal{B}(I)^{\prime}$ in which $a$ is a unit are localizations of $R$; and so $S=\bigcup_{n=1}^{\infty}\left(I^{n} S \cap R\right) / a^{n}=\bigcup_{n=1}^{\infty}\left(a^{n} S \cap R\right) / a^{n}$ and $T=\bigcup_{n=1}^{\infty}\left(a^{n} T \cap R\right) / a^{n}$. Thus there is an element $t$ of $T-S$ having the form $t=b / a^{n}$ for some $b$ in $R$; and hence $b=a^{n} t \in\left(a^{n} T \cap R\right)-\left(a^{n} S \cap R\right)$. Hence $J:=I^{n} \mathcal{B}(K) \cap R>\widetilde{I^{n}}$, which implies that $\mathcal{B}(I) \prec \mathcal{B}(J) \preceq \mathcal{B}(K)$ by [HJLS, Proposition 1.13(b)]. This contradicts our assumption above on $I$.

\section{First and higher coefficient domains.}

(3.1) By (2.3), only a few of the local domains in the blowup of an ideal are necessary to determine the contraction of the extension to an ideal to 
the blowup; we give names to the intersections of those "important" local domains:

Definition 3.2. Suppose $I$ is an ideal primary for the maximal ideal of a $d$ dimensional local domain $R$ and $k \in\{1, \ldots, d\}$. We define the $k$-th coefficient domain $D_{k}$ of $I$ to be the intersection of the local domains on the blowup $\mathcal{B}(I)$ of dimension at most $k$ in which the maximal ideal is an associated prime of the extension of $I$. Thus, under the hypotheses of $(2.11), I D_{k} \cap R=$ $I \mathcal{B}(I)^{(k)} \cap R=I_{\{k\}}$.

(3.3) Since $R$ has infinite residue field by our hypotheses in (2.11), we can choose an element $a$ of $I$ so that each of the local domains on $\mathcal{B}(I)$ in which the maximal ideal is an associated prime of the extension of $I$ is a localization of the same "affine piece of the blowup" $R[I / a]$. Therefore the $k$-th coefficient domain $D_{k}$ of $I$ is a localization of $R[I / a]$ at the complement of finite union of prime ideals of height at most $k$ and hence is a semilocal domain of dimension at most $k$. (Indeed, given a finite set of ideals $J_{1}, \ldots, J_{n}$ having a common reduction $I$, we can choose the element $a$ of $I$ so that, for each $i$ in $\{1, \ldots, n\}$, the $k$-th coefficient domain of $J_{i}$ is a localization of $R\left[J_{i} / a\right]$.) The associated primes of the extension of $I$ of height at most $k$ are the same local domains on each of the models $\mathcal{B}(I), \mathcal{B}(I)^{(k)}$ and $\mathcal{B}(I)^{(k, I)}$, so by $(2.3), I_{\{k\}}=I D_{k} \cap R=I \mathcal{B}(I)^{(k)} \cap I=I \mathcal{B}(I)^{(k, I)} \cap R$.

(3.4) Since $I$ and the powers of $I$ have the same blowup, $I$ and each of its powers have the same $k$-th coefficient domain for each $k$ in $\{1, \ldots, d\}$. It is a consequence of Theorem 3.17 and Proposition 3.2 in [HJLS] that, if $J$ is an ideal such that $I \subseteq J \subseteq I_{\{k\}}$, then $I$ and $J$ have the same $k$-th coefficient domain. 
The $k$-th coefficient domain that we have found most useful is the first; so the remainder of this section is devoted to first coefficient domains.

(3.5) The models $\mathcal{B}(I)$ and $\mathcal{B}(I)^{(1)}$ are complete over $R$, so the Rees valuation domains of $I$ have centers on these models. Moreover, the Rees valuation domains of $I$ are the 1-dimensional local domains of the derived normal model $\mathcal{B}(I)^{\prime}$ that dominate $R$. Each minimal prime of $I \mathcal{B}(I)$ is the contraction of a minimal prime of $I \mathcal{B}(I)^{\prime}$, and since $R$ is quasi-unmixed (so that the dimension formula holds between any affine piece of $\mathcal{B}(I)$ and its integral closure), each minimal prime of $I \mathcal{B}(I)^{\prime}$ contracts to a minimal prime of $I \mathcal{B}(I)$. Thus, the Rees valuation domains of $I$ are obtained by taking integral closure of the first coefficient domain of $I$ and localizing at maximal ideals. The next result follows immediately.

Proposition 3.6. The number of minimal primes of $I \mathcal{B}(I)$ is less than or equal to the number of Rees valuation domains of I. In particular, if I has only one Rees valuation domain, then the first coefficient domain of I is local, i.e., it is itself the unique minimal prime of $I \mathcal{B}(I)$.

(3.7) We can describe the minimal primes of $I \mathcal{B}(I)$, and hence the first coefficient domain of $I$, in terms of the Rees algebra $R[I t]$ (respectively, the extended Rees algebra $\left.R\left[t^{-1}, I t\right]\right)$ of $I$ ( $t$ is an indeterminate): Let $\mathbf{p}$ be a minimal prime of $I R[I t]$ (respectively, of $t^{-1} R\left[t^{-1}, I t\right]$ ); then the intersection $V$ of the localization $R[I t]_{\mathbf{p}}$ (respectively, $R\left[t^{-1}, I t\right]_{\mathbf{p}}$ ) with the field of fractions of $R$ is a minimal prime of $I \mathcal{B}(I)$. And every minimal prime of $I \mathcal{B}(I)$ can be obtained in this way, since we can recover the localizations of the Rees algebra at minimal primes of $I R[I t]$ (respectively of $t^{-1}$ ) from the minimal primes of $I \mathcal{B}(I): V(t)=R[I t]_{\mathbf{p}}$ (respectively, $V(t)=R\left[t^{-1}, I t\right]_{\mathbf{p}}$ ). 
General Example 3.8. Let $I=\left(a_{1}, \ldots, a_{m}\right) R$ be an ideal of $R$ where the elements $a_{1}, \ldots, a_{m}$ are independent in the sense of Kunz [Ku, Definition 4.13, page 144]. Since the kernel of the $R$-algebra homomorphism of the polynomial ring $R\left[x_{1}, \ldots, x_{m}\right]$ to the Rees algebra $R\left[a_{1} t, \ldots, a_{m} t\right]$ taking $x_{i}$ to $a_{i} t$ is contained in $\operatorname{rad}\left(\operatorname{IR}\left[x_{1}, \ldots x_{m}\right]\right)$, it follows that the minimal primes of $I \mathcal{B}(I)$ are in one-to-one correspondence with the minimal primes of $I$. In particular, if $I$ is generated by independent elements and $\operatorname{rad}(I)$ is a prime ideal of $R$, then $I \mathcal{B}(I)$ has only one minimal prime. For example, if $(R, \mathbf{m})$ is 2-dimensional Cohen-Macaulay and $I=(a, b) R$ is a 2-generated m-primary ideal of $R$, then $I \mathcal{B}(I)$ has a unique minimal prime, namely, $R[a / b]_{\mathbf{m} R[a / b]} \cong R(t) /(b t-a)$, the first coefficient domain of $I$.

For a specific example, let $n$ be a positive integer, and in $R=k[x, y]_{(x, y)}$ let $I=\left(x^{n}, y^{n}\right) R$. Then for $t$ an indeterminate,

$$
k\left(y^{n} / x^{n}\right)[x, y]_{(x, y)} \cong R(t) /\left(x^{n} t-y^{n}\right)
$$

is the first coefficient domain $D$ of $I$. Note that $D$ is a complete intersection (and hence Gorenstein) of multiplicity $n$, and that $D$ has the m-adic (or "ord") valuation domain as its integral closure.

(3.9) If each of $I \mathcal{B}(I)$ and $J \mathcal{B}(J)$ has a unique minimal prime, say $D$ and $E$ respectively, and if $D^{\prime} \neq E^{\prime}$, then $I J \mathcal{B}(I J)$ has two distinct minimal primes. (For, if $V$ is a Rees valuation domain of $I$ that dominates $D$ but does not contain $E^{\prime}$, then the center of $V$ on $\mathcal{B}(I J)$ is a minimal prime of $I J \mathcal{B}(I J)$ that does not dominate $E$.) If each of $I, J$ has a unique Rees valuation domain, and if these Rees valuation domains are not the same, then $I J \mathcal{B}(I J)$ has precisely two minimal primes.

(3.10) Let $I \subseteq J$ be ideals primary for the maximal ideal of $R$, with $J$ 
integral over $I$, and let $D$ and $E$ be the first coefficient domains of $I$ and $J$ respectively. Then $D \subseteq E \subseteq D^{\prime}$; also, $I$ and $J$ have the same Rees valuation domains, and $D^{\prime}=E^{\prime}$ is the intersection of the Rees valuation domains of $I$ and $J$. Since $I_{\{1\}}=I D \cap R$ and $J_{\{1\}}=I E \cap R$, it is clear that if $D=E$, then $I_{\{1\}}=J_{\{1\}}$. Conversely, if $I_{\{1\}}=J_{\{1\}}$, then $D=E\left(\right.$ since $I$ and $I_{\{1\}}$ have the same first coefficient domain, and similarly for $J$ ).

Question 3.11. Is the first coefficient domain $D$ associated to an ideal $I$ unique in the following sense: if $E$ is a 1-dimensional semilocal domain birationally containing $R$ such that $I^{n} E \cap R=\left(I^{n}\right)_{\{1\}}$ for all $n$, does it follow that $E \subseteq D ?$

Theorem 3.12. Suppose $(R, \mathbf{m})$ is a normal, analytically unramified quasiunmixed local domain with $R / \mathbf{m}$ infinite and $I$ is an $\mathbf{m}$-primary ideal of $R$. Let $D$ be the first coefficient domain of $I$, and let $E$ be an integral domain between $D$ and the integral closure $D^{\prime}$ of $D$. Then $E$ is the first coefficient domain of an ideal integral over a power of I.

Proof. Assume by way of contradiction that there does not exist such an ideal $J$. The family of domains between $D$ and $E$ satisfies the ascending chain condition, so by replacing $I$ by an ideal integral over one of its powers, we may assume that $D$ is maximal in $E$ with respect to the property of being the first coefficient domain of an ideal integral over a power of $I$.

Let $J_{n}=I^{n} E \cap R$. Since $D^{\prime}$ is the intersection of the Rees valuation domains of $I, I^{n} D^{\prime} \cap R=\left(I^{n}\right)^{\prime}$ for each positive integer $n$. Hence $I^{n} \subseteq$ $J_{n} \subseteq\left(I^{n}\right)^{\prime}$. Since $J_{n}$ is contracted from $E$ and $J_{n} E=I^{n} E$ is principal, $E$ contains the first coefficient domain of $J_{n}$. There exists $a \in I$ such that $I D=a D$. Let $S=R[1 / a] \cap D$ and $T=R[1 / a] \cap E$. Then since we 
can distribute localization over these finite intersections, $D$ and $E$ are the localizations of $S$ and $T$ respectively at the complement of the union of the minimal primes of $a S$ and $a T$. Since $D<E$, we have $S<T$. Let $t \in T-S$. Then $t=b / a^{n}$, where $b \in R$ and $b \in a^{n} T \cap R=a^{n} E \cap R=J_{n}$, but $b \notin a^{n} S \cap R=a^{n} D \cap R=\left(I^{n}\right)_{\{1\}}$. Therefore the first coefficient domain of $J_{n}$ properly contains $D$. This contradiction to the choice of $D$ completes the proof.

(3.13) It is not true that the family of minimal primes of $J \mathcal{B}(J)$ for various ideals $J$ gives $I_{\{1\}}$, for a particular $I$, in the same way that the Rees valuation domains of various $J$ give $I^{\prime}$; i.e., $I^{\prime}=\bigcap\{I V \cap R\}$ where $V$ varies over the Rees valuation domains of various $J$, but $\bigcap\{I S \cap R\}$, where $S$ varies over the minimal primes of various $J \mathcal{B}(J)$, need not be $I_{\{1\}}$. Indeed, if $(R, \mathbf{m})$ is a 2-dimensional Cohen-Macaulay local domain, then any m-primary ideal of $R$ is contracted from a minimal prime in the blowup of an ideal: If $(a, b) R$ is a reduction of the m-primary ideal $I$, then $I=I R[a / b]_{\mathbf{m} R[a / b]} \cap R$ and $R[a / b]_{\mathbf{m} R[a / b]}$ is the unique minimal prime of the blowup of the ideal $(a, b) R$.

Example 3.14. There can exist two (or more) local first coefficient domains $D, E$ with the same integral closure and the same multiplicity and yet $D \neq E$ : Suppose $R=k[x, y]_{(x, y)}$, consider the two minimal reductions $I=\left(x^{2}, y^{2}\right) R$ and $J=\left(x^{2}+y^{2}, x y\right) R$ of $\mathbf{m}^{2} \mathcal{B}(\mathbf{m})$, and let $D, E$ be the first coefficient domains of $I, J$ respectively. Then the residue field of $D$ is $k\left((y / x)^{2}\right)$, while that of $E$ is $k\left(\left(x^{2}+y^{2}\right) / x y\right)$.

(3.15) Assume that $\left\{D_{i}\right\}$ is the set of minimal primes of the extension to its blowup of an $\mathbf{m}$-primary ideal $I$ of a local domain $(R, \mathbf{m})$ with $R / \mathbf{m}$ infinite, and let $D=\bigcap_{i=1}^{n} D_{i}$ be the first coefficient domain of $I$. We would 
like to relate the Hilbert polynomial and the multiplicity of $I$ to those of the extensions of $I$ to $D$ and to the $D_{i}$. If an ideal $J$ of $R$ is generated by a system of parameters, then by General Example 3.8 and [Ku, Corollary 4.19, page 147$], J \mathcal{B}(J)$ has only one minimal prime, say $E$. Moreover, by [Ka, Theorem 1.1] the multiplicity $e(J)$ of $J$ is equal to the multiplicity $e(J E)$ of $J E$. Since the residue field $R / \mathbf{m}$ is infinite, any $\mathbf{m}$-primary ideal $I$ has a reduction $J$ such that $J$ is generated by a system of parameters. Since $J E$ is principal, we have $e(I)=e(J)=e(J E)=\lambda_{E}(E / J E)=\lambda_{E}(D / J D)=$ $\lambda_{E}(D / I D)$. Denoting by $k_{i}$ the dimension of the residue field of $D_{i}$ as an extension of the canonical image of the residue field of $E$ in that of $D_{i}$, and using [ZS, Corollary 1, page 299], we have the following equation relating $e(I)$ to $e\left(I D_{i}\right)$ :

$$
e(I)=e\left(I D_{1}\right) k_{1}+\cdots+e\left(I D_{n}\right) k_{n}
$$

\section{First coefficient ideals and ideals of reduction number at most}

one.

(4.1) Let $I$ be an $\mathbf{m}$-primary ideal in a Cohen-Macaulay local domain $(R, \mathbf{m})$ with $R / \mathbf{m}$ infinite. If $I$ has reduction number at most one, i.e., if $I^{2}=J I$ for some minimal reduction $J$ of $I$, then all powers of $I$ are $e_{1}$-ideals. One way to see this is as follows: By [V, Lemma 1 and Theorem 1], the graded ring $\mathrm{G}(I)$ of $I$ is Cohen-Macaulay, so the extended Rees algebra $R\left[t^{-1}, I t\right]$ is Cohen-Macaulay, so $\mathcal{B}(I)$ is also Cohen-Macaulay. Since $\mathrm{G}(I)$ is CohenMacaulay, all powers of $I$ are contracted from $\mathcal{B}(I)$ [HLS, (1.2)], and since $\mathcal{B}(I)$ is Cohen-Macaulay, all powers of $I$ are $e_{1}$-ideals [HJLS, Corollary 3.11].

(4.2) Under the hypotheses of (4.1), if $I$ has reduction number at most one, i.e., $I^{2}=J I$ for some minimal reduction $J$ of $I$, then this equation holds for 
every minimal reduction of $I$ [Hc, Theorem 2.1]. If in addition $\operatorname{dim}(R)=2$ and $I$ has reduction number at most one, then any power of $I$ has reduction number at most one. (Proof: From the equation $(a, b) I=I^{2}$ we conclude that $\left(I^{n}\right)^{2}=(a, b)^{2 n-1} I \subseteq\left(a^{n}, b^{n}\right) I^{n}$, which implies that $\left(I^{n}\right)^{2}=\left(a^{n}, b^{n}\right) I^{n}$ as required.)

In this section, we further explore the similarities and relationship between the concepts of ideals of reduction number at most one and $e_{1}$-ideals. We begin with a sequence of results to show that, under reasonable hypotheses, both classes are closed under intersection.

Proposition 4.3. Let $I$ be an ideal generated by a regular sequence in a Noetherian ring. Then the set of ideals $J$ for which $I \subseteq J$ and $I J=J^{2}$ is closed under finite intersection. If this set satisfies the descending chain condition, e.g., if $I$ is generated by a system of parameters in a CohenMacaulay local ring, then it is closed under arbitrary intersection.

Proof. Let $J, K$ be two ideals in this set; it suffices to show that $I(J \cap K)=$ $(J \cap K)^{2}$, and for this it is enough to take $f, g$ in $J \cap K$ and show that $f g \in I(J \cap K)$. Let $a_{1}, \ldots, a_{r}$ be a regular sequence that generates $I$; then since $I J=J^{2}$ and $I K=K^{2}$, we can find elements $x_{1}, \ldots, x_{r}$ in $J$ and $y_{1}, \ldots, y_{r}$ in $K$ so that

$$
\begin{aligned}
& a_{1} x_{1}+\cdots+a_{r} x_{r}=f g \\
& a_{1} y_{1}+\cdots+a_{r} y_{r}=f g
\end{aligned}
$$

Subtraction yields

$$
a_{1}\left(x_{1}-y_{1}\right)+\cdots+a_{r}\left(x_{r}-y_{r}\right)=0 \quad,
$$


and since $a_{1}, \ldots, a_{r}$ is a regular sequence, each $x_{i}-y_{i}$ must be in the ideal $I$ generated by $a_{1}, \ldots, a_{r}$ [Ma, Lemma 1 , page 96]. Thus $x_{i}=\left(x_{i}-y_{i}\right)+y_{i} \in$ $J \cap(I+K)=J \cap K$.

Corollary 4.4. Suppose $(R, \mathbf{m})$ is a Cohen-Macaulay local ring with infinite residue field $R / \mathbf{m}$ and $I$ is an $\mathbf{m}$-primary ideal. If the family of ideals integral over I and having reduction number at most one is nonempty, then it has a unique minimal element.

Proposition 4.5. Let $(R, \mathbf{m})$ be a quasi-unmixed analytically unramified local domain with $R / \mathbf{m}$ infinite, and let $I$ be an $\mathbf{m}$-primary ideal. Then the set of $e_{1}$-ideals containing and integral over $I$ is closed under intersection.

Proof. Since the family of all ideals containing $I$ satisfies the descending chain condition, it is enough to show that, if $J_{1}, J_{2}$ are $e_{1}$-ideals containing and integral over $I$, then $J=J_{1} \cap J_{2}$ is also an $e_{1}$-ideal; i.e., that $J$ is contracted from its first coefficient ideal $E$. Let $D, E_{1}, E_{2}$ be the first coefficient domains of $I, J_{1}, J_{2}$ respectively, and let $a$ be an element of $I$ for which $I D=a D$. Then since $E_{1}, E_{2}$ contain and are integral over $E$, which in turn contains and is integral over $D$, we have

$$
J E=a E \subseteq a\left(E_{1} \cap E_{2}\right)=a E_{1} \cap a E_{2}=J_{1} E_{1} \cap J_{2} E_{2}
$$

and since $J_{1}, J_{2}$ are contracted from $E_{1}, E_{2}$ respectively, we have

$$
J \subseteq J E \cap R \subseteq\left(J E_{1} \cap J_{2} E_{2}\right) \cap R=J_{1} \cap J_{2}=J
$$

which completes the proof.

(4.6) Suppose $(R, \mathbf{m})$ is a Cohen-Macaulay local ring with infinite residue field and $I$ is an $\mathbf{m}$-primary ideal. If $\operatorname{dim}(R) \geq 2$ and if the element $a$ of $I$ 
is superficial for $I$, then $I$ has reduction number at most one iff the image of $I$ in $R / a R$ does. This is clear from [Hu, Theorem 2.1] in view of the fact that the Hilbert coefficients $e_{0}$ and $e_{1}$ are unchanged in passing from $I$ to its image in $R / a R$. Thus, the following lemma, essentially set in dimension 1 , will be helpful to us in dimension 2 . We call an ideal $I$ (in a commutative ring with unity) that contains a nonzerodivisor stable iff it has an element $a$ such that $I^{2}=a I$ (i.e., iff it has a principal reduction and reduction number at most one).

Lemma 4.7. Let $D$ be a Noetherian domain of which the integral closure $D^{\prime}$ is a finitely generated $D$-module, and let $\mathbf{q}$ be an ideal of $D$ that is integral over a principal ideal generated by an element of the conductor $\mathbf{c}$ of $D^{\prime}$ into D. Then the Ratliff-Rush ideal $\widetilde{\mathbf{q}}$ associated to $\mathbf{q}$ is stable.

Proof. Since $\widetilde{\mathbf{q}}$ is contracted from its blowup $E$, which is a subring of $D^{\prime}$, it is also contained in c, so we may assume that $\mathbf{q}$ is Ratliff-Rush. Suppose $\mathbf{q}$ is integral over $c D$ where $c \in \mathbf{c}$; then $\mathbf{q}=\mathbf{q} E \cap D=c E \cap D=c E$, so $\mathbf{q}^{2}=(c E)^{2}=c^{2} E=c \mathbf{q}$.

Theorem 4.8. Let $(R, \mathbf{m})$ be a 2-dimensional Cohen-Macaulay analytically unramified local domain with $R / \mathbf{m}$ infinite; let $I=(a, b) R$ be an $\mathbf{m}$-primary ideal, with first coefficient domain $D=R(t) /(b t-a)$; let $J$ be an $e_{1}$-ideal integral over $I$, and let $E$ be the first coefficient domain of J. Then:

(a) E is the blowup of JD;

(b) $e_{0}(J)=e_{0}(J D)$ and $e_{1}(J)=e_{1}(J D)=\lambda_{D}(E / D)$;

(c) $J$ has reduction number at most one iff $J D$ is stable; and

(d) if $J D$ is contained in the conductor of $D^{\prime}$ into $D$, then $J D$ is stable iff $J D$ is a Ratliff-Rush ideal. 
Proof. Both $\operatorname{Spec}(E)$ and $\mathcal{B}(J D)$ consist of the local domains containing $D$ minimal with respect to domination among those in which the extension of $J$ is principal; so (a) holds. Note that since the extension of $J$ to each of these local rings is principal and integral over the extension of $I D=b D$, we have $J E=b E$. Since $b t-a$ is superficial for $J R(t)$, the first assertions of (b) are well known; and if we choose $n$ sufficiently large that $E=J^{n} / b^{n}$, then we see that $e_{1}(J D)=\lambda\left(D / I^{n} D\right)-\lambda\left(D / J^{n} D\right)=\lambda\left(J^{n} D / I^{n} D\right)=\lambda\left(b^{n} E / b^{n} D\right)=$ $\lambda(E / D)$. The implication in (c) that, if $I J=J^{2}$, then $b J D=(J D)^{2}$ is clear; so suppose $J D$ is stable. Then $e_{0}(J)-e_{1}(J)=e_{0}(J D)-e_{1}(J D)=$ $\lambda_{D}(D / J D)=\lambda_{R(t)}(R(t) / J R(t))=\lambda_{R}(R / J)$, so $J$ has reduction number at most one by [Hu, Theorem 2.1]. For (d), if $J D$ is stable, then $J D$ is always Ratliff-Rush by [HLS, (1.1)]; and the converse follows from Lemma 4.7.

(4.9) If $f: R \rightarrow S$ is a surjective ring homomorphism of Noetherian rings, then the preimage in $R$ of an integrally closed ideal in $S$ is integrally closed in $R$. Also if $J$ is a Ratliff-Rush ideal in $S$ and $f^{-1}(J)=I$ contains a regular element of $R$, then $I$ is a Ratliff-Rush ideal of $R$. We observe in Example 4.10 that the corresponding statement for $e_{1}$-ideals fails: if $\mathbf{p}$ is a height- 1 prime of a 2-dimensional $\operatorname{RLR}(R, \mathbf{m})$ and $J$ is an $\mathbf{m}$-primary ideal in $R$ such that $\mathbf{p} \subset J$, it may happen that $J / \mathbf{p}$ is an $e_{1}$-ideal in $R / \mathbf{p}$, but $J$ is not an $e_{1}$-ideal in $R$.

Example 4.10. Let $R=k[[x, y]], I=\left(x^{4}, y^{4}\right) R$, and $S=k\left[\left[t^{4}, t^{5}\right]\right]=R /\left(x^{4}-\right.$ $\left.y^{5}\right) R$, where $x \mapsto t^{5}$ and $y \mapsto t^{4}$. Then the image of $I$ in $S$ is $t^{16} S$, with integral closure $t^{16} k[[t]]$. Let $J=\left(t^{16}, t^{18}, t^{19}\right) S$. Then $J$ is an $e_{1}$-ideal of $S$, but the preimage of $J$ in $R$ is $\left(x^{4}, x^{3} y, x^{2} y^{2}, y^{4}\right) R$, which is properly contained in its $e_{1}$-closure $(x, y)^{4} R$. 
Proposition 4.11. Suppose $(S, \mathbf{n})$ is a Cohen-Macaulay local ring of dimension $d$ for which $S / \mathbf{n}$ is infinite and $I=\left(a_{1}, \ldots, a_{d}\right) S$ is $\mathbf{n}$-primary. Suppose $(I: \mathbf{n})$ is integral over $I$. Then $(I: \mathbf{n})$ has reduction number at most one.

Proof. We write $J$ for $(I: \mathbf{n})$. In view of (4.6), by passing modulo elements of $I$ superficial for $J$, we can reduce to the case where $S$ is a 1-dimensional Cohen-Macaulay local ring. In this case $I=a S$ and $J=(a S: \mathbf{n})=a\left(\mathbf{n}^{-1}\right)$, where the inverse is taken in the total quotient ring of $S$. Since we are assuming that $J$ is integral over $a S$, the ring $S$ is not a DVR. Hence $\mathbf{n n}^{-1}=$ $\mathbf{n}$, so $\left(\mathbf{n}^{-1}\right)^{2}=\mathbf{n}^{-1}$ and therefore $J^{2}=a^{2}\left(\mathbf{n}^{-1}\right)^{2}=a\left(a \mathbf{n}^{-1}\right)=I J$.

(4.12) Suppose $R$, in addition to the hypotheses of (2.11), is CohenMacaulay of dimension 2. We want to explore the consequences of two ideals sharing the same blowup or the same first coefficient domain. Let $J_{1}, J_{2}$ be m-primary $e_{1}$-ideals, and assume that $J_{1}$ has reduction number at most one. If they share a common blowup and have powers $J_{1}^{m}, J_{2}^{n}$ that share a common reduction $I$, then we may assume that the exponents are sufficiently large that both powers are Ratliff-Rush ideals. Then they are both the contraction of the extension of $I$ to their common blowup and hence are equal; so by [Sa, Corollary 2.6] and [Hu, Theorem 2.1], $e_{2}\left(J_{2}\right)=e_{2}\left(J_{2}^{m}\right)=e_{2}\left(J_{1}^{n}\right)=0$, and $J_{2}$ has reduction number at most one. We can replace the assumption that they share a common blowup with the assumptions that they share a common first coefficient domain and that $\mathcal{B}\left(J_{2}\right)$ is Cohen-Macaulay (this is always true if Question 2.12(3) has a positive answer), and the same argument shows that $J_{2}$ has reduction number at most one. But if we assume only that they have a common first coefficient domain, or if we remove the 
assumption that they have powers with a common reduction, we do not see that the property of reduction number at most one transfers from one to the other.

\section{In a two-dimensional regular local ring.}

(5.1) In this section we provide general and specific examples, in the context of a 2-dimensional RLR, of the concepts we have introduced earlier. We begin by noting that we can give meaning to the concept of coefficient ideals that are not primary for the maximal ideal in a local domain, but in a 2-dimensional RLR, the study of coefficient ideals reduces to the $\mathbf{m}$-primary case.

(5.2) Let $I$ be an ideal in a Noetherian domain $D$ of dimension $d$ and $m \in\{1, \ldots, d\}$. With the motivation of Theorem 3.17 of [HJLS], in [HJLS, Definition 3.21] the definition of the $e_{m}$-ideal $I_{\{m\}}$ associated with $I$ is extended to the case where $D$ is not local or $I$ is not primary for the maximal ideal of $D: I_{\{m\}}:=I \mathcal{B}(I)^{(m, I)} \cap D$. Now it is shown in [HLS, (1.10)] that $I=a J$ does not in general imply $\widetilde{I}=a \widetilde{J}$. But this misbehavior does not occur in integrally closed domains, e.g., RLRs [HLS, (1.12)(ii)]. Similarly, in an integrally closed domain $D$ the corresponding fact holds for all associated $e_{m}$-ideals: For $I$ an ideal in $D, a$ in $R-0$ and $m<d$, if $x \in(a I)_{\{m\}}=(a I) \mathcal{B}(a I)^{(m, I)} \cap D$, then

$$
\begin{aligned}
x / a & \in \bigcap\left\{I S: S \in \mathcal{B}(I)^{(m, I)}\right\} \subseteq \bigcap\left\{S: S \in \mathcal{B}(I)^{(m, I)}\right\} \\
& \subseteq \bigcap \text { valuation domains between } D \text { and its field of fractions }\}=D,
\end{aligned}
$$

so $x \in(a I) \mathcal{B}(I)^{(m, I)} \cap a D=a\left(I_{\{m\}}\right)$; since the reverse inclusion always holds, we have $(a I)_{\{m\}}=a\left(I_{\{m\}}\right)$. Thus, it is immediate that the result corresponding to [HLS, (1.12)(iii)] is valid for all the associated $e_{m}$-ideals 
with the above definition: If $I=a J$ and $I=I_{\{m\}}$, then $J=J_{\{m\}}$. Since every nonzero ideal of a 2 -dimensional $\operatorname{RLR}(R, \mathbf{m})$ is a principal multiple of an m-primary ideal and the blowups of these two isomorphic ideals are the same, the study of associated $e_{1}$-ideals for arbitrary nonzero ideals $I$ reduces to that of $\mathbf{m}$-primary ideals. More precisely, we have $I=a J$, where $J$ is an m-primary ideal; if one of $I, J$ is Ratliff-Rush, then so is the other, and if one is an $e_{1}$-ideal, then so is the other.

(5.3) In a 2-dimensional RLR $(R, \mathbf{m})$, let $x, y$ be a regular system of parameters, let $J$ be an ideal with integral closure a power $\mathbf{m}^{n}$ of $\mathbf{m}$, let $I=(a, b) R$ be a minimal reduction of $J$, and let $D=R(t) /(b t-a) R(t)$ be the first coefficient domain of $I$. Then we claim that $J D$ is contained in the conductor c of $D^{\prime}=V$, the ord-valuation domain, into $D$. Since $J$ is contained in $\mathbf{m}^{n}$, to prove the claim it is enough to show that $\mathbf{m}^{n-1} D^{\prime} \subseteq \mathbf{c}$ : The residue fields of $D, V$ are $k(a / b), k(x / y)$ respectively, and the maximal ideal of $V$ is the extension of that of $D$; so the length of a $V$-module as a $D$-module is the degree of the residue field extension $[k(x / y): k(a / b)]$ times its length as a $V$-module. Since $b t-a \in I R(t)$, we have

$$
\begin{aligned}
n^{2} & =\lambda_{R}(R / I)=\lambda_{R(t)}(R(t) / I R(t))=\lambda_{D}(D / I D) \\
& =\lambda_{D}(D / b D)=\lambda_{D}(V / b V)+\lambda_{D}(b V / b D)-\lambda_{D}(V / D)=\lambda_{D}(V / b V) \\
& =\operatorname{ord}(b) \cdot[k(x / y): k(a / b)]=n \cdot[k(x / y): k(a / b)],
\end{aligned}
$$

so $[k(x / y): k(a / b)]=n$. Thus, the generator $x / y$ of $k(x / y)$ over $k(a / b)$ satisfies a monic polynomial of degree $n$ over the smaller field; so by Nakayama's lemma, the elements $1, x / y,(x / y)^{2}, \ldots,(x / y)^{n-1}$ form a $D$-module basis for $V$. Thus, $y^{n-1} \in \mathbf{c}$, and $y^{n-1}$ generates $\mathbf{m}^{n-1} V$.

It follows by Theorem 4.8 that an $e_{1}$-ideal in a 2 -dimensional RLR whose 
integral closure is a power of the maximal ideal has reduction number at most one iff its extension to its first coefficient domain is Ratliff-Rush. Example 5.4 shows that these conditions need not hold.

Example 5.4. In $R=k[x, y]_{(x, y)}$, let $J=\left(x^{7}, y^{7}, x^{5} y^{3}+x^{3} y^{5}, x^{6} y^{4}\right) R$. Then $H_{J}(n)=49\left(\begin{array}{c}n+1 \\ 2\end{array}\right)-10\left(\begin{array}{c}n \\ 1\end{array}\right)+1$, so $R[J t]$ is not Cohen-Macaulay and $J$ has reduction number greater than one. Using the computer program MACAULAY, written by David Bayer and Michael Stillman, we find that $J$ has reduction number 2 and that a minimal projective resolution of $R[I t]$ as a module over the polynomial ring $S$ in 4 variables over $R$ has the form

$$
0 \longrightarrow S^{1} \stackrel{\varphi_{4}}{\longrightarrow} S^{7} \stackrel{\varphi_{3}}{\longrightarrow} S^{13} \stackrel{\varphi_{2}}{\longrightarrow} S^{8} \stackrel{\varphi_{1}}{\longrightarrow} S^{1} \stackrel{\varphi_{0}}{\longrightarrow} R[I t] \longrightarrow 0
$$

and the entries of the matrix $\varphi_{4}$ generate the homogeneous maximal ideal of $S$. Therefore, from the Auslander-Buchsbaum formula [Ma, page 114] it follows that $\operatorname{depth}(R[I t])=2$; and if we homogeneously localize at any of the homogeneous primes containing $\operatorname{ker} \varphi_{0}$ other than the homogeneous maximal ideal of $S$, the projective dimension decreases. Again, using the Auslander-Buchsbaum formula, it follows that the resulting localizations of $R[I t]$ are Cohen-Macaulay; i.e., $\mathcal{B}(I)$ is Cohen-Macaulay. Also, since $R[I t]$ is not Cohen-Macaulay, neither is the associated graded ring $\mathrm{G}(I)$ [HM, Proposition 2.6] [JV, Theorem 4.1] [Sh2, Corollary 4(f)]; so by [HM, Theorem 2.1] we have $\operatorname{depth}(\mathrm{G}(I))=\operatorname{depth}(R[I t])-1>0$. By [HLS, (1.2)] all powers of $J$ are contracted from $\mathcal{B}(J)$ and hence [HJLS, Corollary 3.11] are $e_{1}$-ideals.

Another example of an ideal $J$ of $R=k[x, y]_{(x, y)}$ having reduction number greater than one, but for which the Rees algebra $R[J t]$ satisfies Serre's $S_{2}$ condition and so $\mathcal{B}(J)$ is Cohen-Macaulay, is 


$$
J=\left(x^{8}, y^{8}, x^{6} y^{3}+x^{3} y^{6}, x^{4} y^{7}\right) R
$$

The Hilbert polynomial of this ideal is $64\left(\begin{array}{c}n+1 \\ 2\end{array}\right)-14\left(\begin{array}{c}n \\ 1\end{array}\right)+1$. For both of these ideals the Hilbert function agrees with its Hilbert polynomial for all positive $n$.

(5.5) Let $(R, \mathbf{m})$ be a 2-dimensional RLR with regular system of parameters $x, y$, let $I=\left(x^{n}, y^{n}\right) R$, and let $J$ be an ideal integral over $I$. By the Northcott inequality, $\lambda(J / I) \leq e_{1}(J)$. Thus, if $e_{1}(J)=0$, then $J$ has reduction number 0 . If $e_{1}(J)=1$, then $J$ has reduction number at most one by Proposition 4.11. If $e_{1}(J)=2$, then (cf. Theorem 4.8) since $\lambda_{D}(J E / I D)=2$, so either $J D=J E$ is stable, and hence $J$ has reduction number at most one, or $\lambda_{D}(J D / I D)=1$, so that again $J$ has reduction at most one by Proposition 4.11. Example 5.4 has $e_{1}(J)=10$. Is it possible to find an $e_{1}$-ideal $J$ that does not have reduction number at most one, but for which $e_{1}(J)<10$ ?

(5.6) We return to the notation of (5.3), and continue that discussion with a general remark: For any 1-dimensional local domain $S$ that birationally dominates $R$ and has integral closure $V$, the multiplicity of $S$ is the degree of the residue field $k(x / y)$ of $V$ over the residue field of $S$. (For, the maximal ideal of $V$ is $x V$, and the multiplicity of $S$ is $\lambda_{S}(S / x S)=\lambda_{S}(V / x V)$, which is the residual degree.) Thus, in particular, $D$ has multiplicity $n$. Also, $D$ has embedding dimension 2. The first coefficient domain of the ideal $J$ contains $D$ and is contained in $D^{\prime}=V$; its multiplicity divides $n$. In Example 5.7, we see that if $n>2$, there can be a first coefficient domain strictly between $D$ and $V$ which also has multiplicity $n$. 
Example 5.7. Let $R=k[x, y]_{(x, y)}$ and $J=\left(x^{6}, x^{5} y, x^{3} y^{2}, y^{5}\right) R$. Then the integral closure $J^{\prime}$ of $J$ is obtained by adjoining to $J$ the monomials $x y^{4}, x^{2} y^{3}$, and the factorization of $J^{\prime}$ into a product of complete simple ideals is $J^{\prime}=(x, y)^{3} R \cdot\left(x^{3}, x^{2} y, y^{2}\right) R$. So the ideal $J$ has two Rees valuation domains, the ord valuation domain $V=R[y / x]_{\mathbf{m} R[y / x]}$ and the DVR $W$ which is the integral closure of $R\left[y^{2} / x^{3}\right]_{\mathbf{m} R\left[y^{2} / x^{3}\right]}$. Consider the affine piece $R\left[x^{3} / y^{2}, x^{2} / y, y^{3} / x^{3}\right]$ of $\mathcal{B}(J)$. Both the Rees valuation domains of $J$ contain this affine piece and are centered on distinct height-one primes of it. Moreover, we see that $W$ is a localization of this domain, for $x^{3} / y^{2}$ is a generator for the residue field of $W$ over $k$ and $x^{2} / y$ is an element of $W$-value one. On the other hand, if $E$ denotes the 1-dimensional local domain of $\mathcal{B}(J)$ dominated by $V$, then we claim that $E$ is not Gorenstein: Let $D=R\left[y^{3} / x^{3}\right]_{\mathbf{m} R\left[y^{3} / x^{3}\right]}$. Note that $D$ has multiplicity 3 and integral closure $D^{\prime}=V=D[y / x]$. Using that $1, x / y, x^{2} / y^{2}$ is a module basis for $V$ over $D$ and hence a module basis of $V$ over $E=D\left[x^{2} / y\right]$, one can check that the conductor of $V$ to $E$ is the maximal ideal $\left(x, y, x^{2} / y\right) E$ of $E$. Therefore the length of $E$ modulo the conductor of $V$ to $E$ is one, while $\lambda_{E}(V / E)=2$, so $E$ is not Gorenstein.

Additional comments about the 1-dimensional local domain $E$ of the above example: If $J_{1}=\left(x^{3}, x^{2} y^{2}, y^{3}\right) R$, then $E$ is also a minimal prime of $J_{1} \mathcal{B}\left(J_{1}\right)$, indeed, the first coefficient domain of $J_{1} \mathcal{B}\left(J_{1}\right)$. For, $J_{1}$ has $V$ as its unique Rees valuation domain, and $R\left[x^{2} / y, x^{3} / y^{3}\right]$ is an affine piece of the blowup of $J_{1}$ for which the localization at the center of the ord valuation is $D\left[x^{2} / y\right]$, where $D=R\left[x^{3} / y^{3}\right]_{\mathbf{m} R\left[x^{3} / y^{3}\right]}$ as above. It is remarked in (E5) of [HJLS, Examples 6.1] that the ideal $J_{1}$ has Hilbert coefficients $e_{0}=9, e_{1}=1, e_{2}=0$ and reduction number one. So all the powers of $J_{1}$ are $e_{1}$-ideals. 
(5.8) Suppose $(R, \mathbf{m})$ is a 2-dimensional RLR and $S$ is a 1-dimensional semilocal domain that birationally dominates $R$ and is a ring of fractions of a finitely generated $R$-algebra. It would be interesting to have necessary and sufficient conditions in order that $S$ is the first coefficient domain of an ideal of $R$. It is well known that the integral closure $S^{\prime}$ of $S$ is the first coefficient domain of an integrally closed $\mathbf{m}$-primary ideal, since it is a finite intersection of DVRs, which are the Rees valuation domains of such an ideal.

\section{ACKNOWLEDGEMENT}

We would like to thank Craig Huneke for his help with the use of MACAULAY and for his interest in our work.

\section{REFERENCES}

[[EGA]]A. Grothendieck and J. Dieudonné, Éléments de Géométrie Algébrique II, Publ. Math. I. H. E. S. 8 (1961).

[[HJLS]William Heinzer, Bernard Johnston, David Lantz and Kishor Shah, Coefficient ideals in and Blowups of a commutative Noetherian domain, J. Algebra (to appear).

[[HLS]] William Heinzer, David Lantz and Kishor Shah, The Ratliff-Rush ideals in a Noetherian ring, Comm. in Algebra 20 (1992), 591-622.

[[Hc]] Sam Huckaba, Reduction numbers for ideals of higher analytic spread, Math. Proc. Camb. Philos. Soc. 102 (1987), 49-57.

[[HM]] S. Huckaba and T. Marley, Depth properties of Rees algebras and associated graded rings, J. Algebra (to appear).

[[Hu]] Craig Huneke, Hilbert functions and symbolic powers, Michigan J. Math. 35 (1987), 293-318.

[[JV]] Bernard Johnston and Jugal Verma, On the length formula of Hoskin and Deligne and associated graded rings of two-dimensional regular local rings, Proc. Cambridge Phil. Soc. (to appear).

[[Ka]] Daniel H. Katz, Note on multiplicity, Proc. Amer. Math. Soc. 104 (1988), 10211026.

[[Ku] Ernst Kunz, Introduction to Commutative Algebra and Algebraic Geometry, Birkhäuser, Boston, 1985.

[[Ma]] Hideyuki Matsumura, Commutative Algebra, Second Edition, Benjamin/Cummings, Reading, Massachusetts, 1980.

[[N]] M. Narita, A note on the coefficients of Hilbert characteristic functions in semiregular local rings, Proc. Camb. Philos. Soc. 59 (1963), 269-275.

[[Re1]] D. Rees, a-Transforms of local rings and theorem on multiplicities of ideals, Proc. Camb. Philos. Soc. 57 (1961), 8-17. 
[[Re2]] D. Rees, A note on analytically unramified rings, J. London Math. Soc. 36 (1961), 24-28.

[[Sa]] Judith Sally, Ideals whose Hilbert function and Hilbert polynomial agree at $n=1$, Preprint.

[[Sh1]] Kishor Shah, Coefficient ideals, Trans. Amer. Math. Soc. 327 (1991), 373-384.

[[Sh2]] Kishor Shah, On the Cohen-Macaulayness of the fiber cone of an ideal, J. Algebra 143 (1991), 156-172.

[[V]] G. Valla, On form rings which are Cohen-Macaulay, J. Algebra 58 (1979), 247250 .

[[ZS]] Oscar Zariski and Pierre Samuel, Commutative Algebra, Volume II, Springer, Berlin, 1975. 